International Journal of Biomedicine I June 2019 - Volume 9, Issue Suppl_1: Abstracts From the Second Russian International Conference "Cryo-electron microscopy 2019: achievements and prospects"

POSTER ABSTRACT PRESENTATIONS

SESSION TITLE: STRUCTURE OF MEMBRANE PROTEINS

DOI: 10.21103/IJBM.9.Suppl_1.P5

\title{
Abstract P-5: Modern Membrane Mimetic Systems for Cryo-EM Based Structural Analysis
}

Egor S. Kolesnikov, Polina A. Khorn, Anastasiia U. Gusach, Aleksandra P. Luginina, Alexey V. Mishin

Research Center for Molecular Mechanisms of Aging and Age-Related Diseases, Moscow Institute of Physics and Technology, Dolgoprudny, Russia

Background: Single particle approach that is used to image the sample by an electron beam requires receptors to be extracted from the cell membrane and placed into the sample grid in the solubilized form. Conventional use of detergents for GPCR solubilization often leads to poor stability of the receptor removed from its natural lipid environment. Therefore, scientists use membrane mimetic systems (MMS) such as amphipols and various types of nanodiscs, including styrene maleic acid (SMA) derivatives - SMALP and DISMALP. The last one are among the most prospective and interesting MMS and could be used as a tool in Cryo-EM studies. SMALP and DIBMALP have better thermal stability than other types of nanodiscs. SMA could negatively affect the order of phospholipids but DIBMA has a weaker hydrophilic part that is thought to be a reason of weaker impact on phospholipids.

Methods: Thermal stability of the nanodiscs was tested using microscale thermophoresis and thermal shift assay.

Results: In this work, adenosine $\mathrm{A}_{2 \mathrm{a}}$ GPCR was solubilized into SMALP and DIBMALP. Then its stability was tested.

Conclusion: SMALP and DIBMALP are useful in Cryo-EM studies. It is better to stabilise membrane proteins in SMALP and DIBMALP for structural analisys because of their high thermal stability.

Key Words: nanodiscs $・$ SMALP $・$ DIBMALP • Cryo-EM

Sources of Funding: This work was supported by the Russian President Grant for Governmental support of Young Scientists (project no. MK-5184.2018.4)

International Journal of Biomedicine. 2019;9 Suppl 1: S18-19. doi: 10.21103/IJBM.9.Suppl_1.P5

C2019 International Medical Research and Development Corporation 